\title{
CONVERGENCE ANALYSIS OF PRECONDITIONED AOR ITERATIVE METHOD
}

\author{
P. Hessari ${ }^{a}$, M. T. DARVIShI ${ }^{b}$, B.-C. Shin ${ }^{a, 1}$
}

\begin{abstract}
In this paper, we consider a preconditioned accelerated overrelaxation (PAOR) method to solve systems of linear equations. We show the convergence of the PAOR method. We also give comparison results when the coefficient matrix is an $L$ - or $H$-matrix. Finally, we provide some numerical experiments to show efficiency of PAOR method.
\end{abstract}

\section{Introduction}

Consider the following linear system of $n$ equations

$$
\mathcal{A x}=\mathbf{b}
$$

where $\mathcal{A}=\left(a_{i j}\right) \in \mathbb{R}^{n \times n}$ is an $n \times n$ nonsingular matrix, and $\mathbf{x}, \mathbf{b} \in \mathbb{R}^{n}$. If $\mathcal{A}$ is splitted into

$$
\mathcal{A}=M-N
$$

where $M$ is a nonsingular matrix, then the basic splitting iterative method can be expressed as:

$$
\mathbf{x}^{(k+1)}=M^{-1} N \mathbf{x}^{(k)}+M^{-1} \mathbf{b}, \quad k=0,1,2, \cdots .
$$

As it is well known, the above iterative method is convergent to the unique solution $x=\mathcal{A}^{-1} \mathbf{b}$ for each initial value $x^{(0)}$ if and only if the spectral radius of the iteration matrix $M^{-1} N$ satisfies $\rho\left(M^{-1} N\right)<1$. To improve the convergence rate of the basic iterative method, several preconditioned iterative methods have been proposed (see, e.g., $[1,2,3]$ ).

Received May 12, 2010. Accepted August 27, 2010.

Key words and phrases: Preconditioner; AOR iterative method; $H$-matrix; $L$-matrix.

[1] Corresponding author. Fax: +82-62-530-3339. Email address: bcshin@jnu.ac.kr.

The author was financially supported by special research program of Chonnam National University, 2009. 
The main idea of these preconditioned iterative methods is to transform the original system into the preconditioned form

$$
P \mathcal{A} \mathbf{x}=P \mathbf{b}
$$

where $P \in \mathbb{R}^{n \times n}$ is a nonsingular matrix. Then the basic iterative scheme of the preconditioned system is given by

$$
\mathbf{x}^{(k+1)}=M_{P}^{-1} N_{P} \mathbf{x}^{(k)}+M_{P}^{-1} \mathbf{b}, \quad k=0,1,2, \cdots,
$$

where $P \mathcal{A}=M_{P}-N_{P}$ with a nonsingular matrix $M_{P}$.

Without loss of generality, suppose that the coefficient matrix $\mathcal{A}$ has the following splitting

$$
\mathcal{A}=\mathcal{I}-\mathcal{L}-\mathcal{U}
$$

where $\mathcal{I}$ is identity matrix, $-\mathcal{L}$ and $-\mathcal{U}$ are strictly lower and upper triangular matrix of $\mathcal{A}$, respectively. For this splitting, the AOR iterative method is as follows:

$$
x^{(i+1)}=L_{r w} x^{(i)}+\omega(\mathcal{I}-r \mathcal{L})^{-1} \mathbf{b}, \quad i=0,1,2, \cdots,
$$

where

$$
L_{r w}=(\mathcal{I}-r \mathcal{L})^{-1}[(1-\omega) \mathcal{I}+(\omega-r) \mathcal{L}+\omega \mathcal{U}]
$$

is the iteration matrix and $r, \omega$ are acceleration parameters with $\omega \neq 0$.

Liu et al. [1] considered $P=I+S_{\beta}$ as a preconditioner and gave the sufficient conditions for convergence of the Gauss-Seidel method when the coefficient matrix $\mathcal{A}$ is an $H$-matrix, where

$$
S_{\beta}=\left(\begin{array}{ccccc}
0 & 0 & \cdots & \cdots & 0 \\
-\beta_{1} a_{21} & 0 & \cdots & 0 & 0 \\
0 & -\beta_{2} a_{32} & \cdots & 0 & 0 \\
\vdots & \vdots & \cdots & \ddots & \vdots \\
0 & 0 & \cdots & -\beta_{n-1} a_{n, n-1} & 0
\end{array}\right)
$$

whereas $\beta_{i} \geq 0, i=1, \cdots, n-1$.

Consider the preconditioned linear system

$$
\mathcal{A}_{\beta} \mathbf{x}=\mathbf{b}_{\beta}
$$

where $\mathcal{A}_{\beta}=\left(I+S_{\beta}\right) A$ and $\mathbf{b}_{\beta}=\left(I+S_{\beta}\right) \mathbf{b}$.

In this paper, we will show the convergence analysis for the preconditioned AOR method when the coefficient matrix $\mathcal{A}$ is an $L$ - or an $H$-matrix. 


\section{Preliminaries}

For convenience, some notations, definitions and some results that will be used in the next sections are given.

A matrix $A$ is called nonnegative(positive) if each entry of $A$ is nonnegative(positive). We denote it by $A \geq 0(>0)$. Similarly, for $n$-dimensional vectors $x$, by identifying them with $n \times 1$ matrices, we can also define $\mathbf{x} \geq 0(>0)$. Denote by $\rho(A)$ the spectral radius of $A$.

Definition 2.1. [6] $A$ real matrix $A$ is called an $M$-matrix if $A=$ $s I-B, B \geq 0$ and $s>\rho(B)$.

Definition 2.2. [7, 9] $A$ matrix $A=\left(a_{i j}\right)$ is called

1. a $Z$-matrix if $a_{i j} \leq 0$ for $i, j=1,2, \cdots, n$ such that $i \neq j$,

2. an $L$-matrix if $a_{i j} \leq 0$ for $i, j=1,2, \cdots, n,(i \neq j)$ and $a_{i i}>0$, $i=1,2, \cdots, n$,

3. an $H$-matrix if its comparison matrix $\langle A\rangle=\left(\bar{a}_{i j}\right)$ is a nonsingular $M$-matrix, where $\bar{a}_{i j}$ is

$$
\bar{a}_{i i}=\left|a_{i, i}\right|, \quad \bar{a}_{i j}=-\left|a_{i j}\right|, \quad i \neq j .
$$

It must be noted that an $L$-matrix $A$ is a nonsingular $M$-matrix if $A$ is nonsingular and $A^{-1} \geq 0$.

Definition 2.3. [7] A matrix $A$ is irreducible if the directed graph associated to $A$ is strongly connected.

Definition 2.4. Let $A$ be a real matrix. The representation

$$
A=M-N
$$

is called a splitting of $A$ if $M$ is a nonsingular matrix. The splitting is said to be

1. convergent if $\rho\left(M^{-1} N\right)<1$;

2. regular if $M^{-1} \geq 0$ and $N \geq 0$;

3. nonnegative if $M^{-1} N \geq 0$;

4. $M$-splitting if $M$ is a nonsingular $M$-matrix and $N \geq 0$.

It is obvious that an $M$-splitting is regular and a regular splitting is nonnegative.

Lemma 2.5. [7] Let $A \geq 0$ be an irreducible $n \times n$ matrix. Then

1. $A$ has a positive real eigenvalue equal to its spectral radius $\rho(A)$,

2. for $\rho(A)$ there corresponds an eigenvector $x>0$, 
3. $\rho(A)$ is a simple eigenvalue of $A$,

4. $\rho(A)$ increases when any entry of $A$ increases.

Lemma 2.6. [6] Let $A$ be a nonnegative matrix.

1. If $\alpha x \leq A x$ for some nonnegative vector $x, x \neq 0$, then $\alpha \leq \rho(A)$.

2. If $A x \leq \beta x$ for some positive vector $x$, then $\rho(A) \leq \beta$.

Moreover, if $A$ is irreducible and if $0 \neq \alpha x \leq A x \leq \beta x$ for some nonnegative vector $x$, then $\alpha \leq \rho(A) \leq \beta$ and $x$ is a positive vector.

Lemma 2.7. [8] Let $A=M-N$ be an $M$-splitting of $A$. Then $\rho\left(M^{-1} N\right)<1$ if and only if $A$ is a nonsingular $M$-matrix.

Theorem 2.8. [6] Let $A$ be a $Z$-matrix. Then the following statements are equivalent:

1. $A$ is nonsingular $M$-matrix.

2. There is a positive vector $x$ such that $A x>0$.

3. All principal submatrices of $A$ are $M$-matrices.

Lemma 2.9. Let $\mathcal{A}$ be a $Z$-matrix. Then $\mathcal{A}$ is a nonsingular $M$ matrix if and only if $\mathcal{A}_{\beta}$ is a nonsingular $M$-matrix for $\beta_{i} \in[0,1], i=$ $1,2, \cdots, n-1$.

Proof. Let $\mathcal{A}$ be a nonsingular $M$-matrix. We have

$$
\begin{aligned}
& \mathcal{A}_{\beta}=\left(I+S_{\beta}\right) \mathcal{A}= \\
& \left(\begin{array}{cccc}
1 & a_{12} & \cdots & a_{1 n} \\
a_{21}-\beta_{1} a_{21} & 1-\beta_{1} a_{21} a_{12} & \cdots & a_{2 n}- \\
\vdots & \vdots & \ddots & \beta_{1} a_{21} a_{1 n} \\
a_{n 1}-\beta_{n-1} a_{n, n-1} a_{n-1,1} & a_{n 2}-\beta_{n-1} a_{n, n-1} a_{n-1,2} & \cdots & \vdots \\
& & & \beta_{n-1} a_{n, n-1} \\
& & & a_{n-1, n}
\end{array}\right) .
\end{aligned}
$$

Suppose that $\mathcal{A}$ is a nonsingular $M$-matrix, then by Theorem 2.8, there exists a positive vector $x$ such that $\mathcal{A} \mathbf{x}>0$. On the other hand, since $\mathcal{A}$ is a $Z$-matrix, $S_{\beta}>0$. So for the above vector $x$, we have $\mathcal{A}_{\beta} \mathbf{x}=\left(I+S_{\beta}\right) \mathcal{A} \mathbf{x}>0$. Hence, by Theorem $2.8, \mathcal{A}_{\beta}$ is a nonsingular $M$ matrix. Note that if $\mathcal{A}_{\beta}$ be an $M$-matrix, then $\mathcal{A}_{\beta}^{T}$ is also an $M$-matrix. By Theorem 2.8, there exists a positive vector $\mathbf{x}$ such that $\mathcal{A}_{\beta}^{T} \mathbf{x}>0$, so $\mathcal{A}^{T}\left(I+S_{\beta}^{T}\right) \mathbf{x}>0$. Set $\mathbf{y}=\left(I+S_{\beta}^{T}\right) \mathbf{x}$. Then, we have $\mathbf{y}>0$ and 
$\mathcal{A}^{T} \mathbf{y}>0$, which means that $\mathcal{A}^{T}$ is a nonsingular $M$-matrix, hence, $\mathcal{A}$ is also a nonsingular $M$-matrix.

\section{The preconditioned AOR method for $L$-matrices}

In this section, we consider the preconditioned linear system

$$
\mathcal{A}_{\beta} \mathbf{x}=\mathbf{b}_{\beta}
$$

where $\mathcal{A}_{\beta}=\left(I+S_{\beta}\right) \mathcal{A}$ and $\mathbf{b}_{\beta}=\left(I+S_{\beta}\right) \mathbf{b}$. We split the coefficient matrix $\mathcal{A}_{\beta}$ as

$$
\mathcal{A}_{\beta}=\mathcal{D}_{\beta}-\mathcal{L}_{\beta}-\mathcal{U}_{\beta}
$$

where $\mathcal{D}_{\beta},-\mathcal{L}_{\beta}$, and $-\mathcal{U}_{\beta}$ are the diagonal, strictly lower and strictly upper triangular matrices of $\mathcal{A}_{\beta}$, respectively. Then the preconditioned AOR iterative method is as follows:

$$
\mathbf{x}^{(i+1)}=\tilde{L}_{r w} \mathbf{x}^{(i)}+\omega\left(\mathcal{D}_{\beta}-r \mathcal{L}_{\beta}\right)^{-1} \mathbf{b}_{\beta}, \quad i=0,1,2, \cdots,
$$

where

$$
\tilde{L}_{r w}=\left(\mathcal{D}_{\beta}-r \mathcal{L}_{\beta}\right)^{-1}\left[(1-\omega) \mathcal{D}_{\beta}+(\omega-r) \mathcal{L}_{\beta}+\omega \mathcal{U}_{\beta}\right]
$$

is the iteration matrix.

Lemma 3.1. Let $\mathcal{A}$ and $\mathcal{A}_{\beta}$ be the coefficient matrices of linear system (1) and (6), respectively. Suppose that $\mathcal{A}$ is irreducible $L$-matrix and $0 \leq r \leq \omega \leq 1(r \neq 1, \omega \neq 0)$.

1. The iterative matrix $L_{r \omega}$ in (5) is a nonnegative irreducible matrix.

2. If there exists a nonempty set $\alpha \subset Q=\{2,3, \cdots, n\}$ such that

$0<a_{i, i-1} a_{i-1, i}<1, \quad i \in \alpha \quad$ and $\quad a_{i, i-1} a_{i-1, i}=0, \quad i \in Q \backslash \alpha$,

then $\tilde{L}_{r w}$ in (7) is a nonnegative irreducible matrix.

Proof. (a) Note that

$$
\begin{aligned}
L_{r w}= & (\mathcal{I}-r \mathcal{L})^{-1}[(1-\omega) \mathcal{I}+(\omega-r) \mathcal{L}+\omega \mathcal{U}] \\
= & \left(\mathcal{I}+r \mathcal{L}+r^{2} \mathcal{L}^{2}+\cdots+r^{n-1} \mathcal{L}^{n-1}\right)[(1-\omega) \mathcal{I}+(\omega-r) \mathcal{L}+\omega \mathcal{U}] \\
= & (1-\omega) \mathcal{I}+(\omega-r) \mathcal{L}+\omega \mathcal{U}+r \mathcal{L}[(1-\omega) \mathcal{I}+(\omega-r) \mathcal{L}+\omega \mathcal{U}] \\
& \quad+\left[r^{2} \mathcal{L}^{2}+\cdots+r^{n-1} \mathcal{L}^{n-1}\right][(1-\omega) \mathcal{I}+(\omega-r) \mathcal{L}+\omega \mathcal{U}] \\
= & (1-\omega) \mathcal{I}+\omega(1-r) \mathcal{L}+\omega \mathcal{U}+T
\end{aligned}
$$

where

$T=r \mathcal{L}[(\omega-r) \mathcal{L}+\omega \mathcal{U}]+\left[r^{2} \mathcal{L}^{2}+\cdots+r^{n-1} \mathcal{L}^{n-1}\right][(1-\omega) \mathcal{I}+(\omega-r) \mathcal{L}+\omega \mathcal{U}] \geq 0$.

Since $\mathcal{A}$ is an $L$-matrix, it holds that $\mathcal{I} \geq 0, \mathcal{L} \geq 0$ and $\mathcal{U} \geq 0$, using the fact that $0 \leq r \leq \omega \leq 1(r \neq 1, \omega \neq 0)$, we have $L_{r \omega} \geq 0$. Since $\mathcal{A}$ is 
an irreducible matrix, so is $(1-\omega) \mathcal{I}+\omega(1-r) \mathcal{L}+\omega \mathcal{U}$. Thus $L_{r w}$ is an irreducible matrix.

(b) Note that

$$
\begin{aligned}
\tilde{L}_{r w} & =\left(\mathcal{D}_{\beta}-r \mathcal{L}_{\beta}\right)^{-1}\left[(1-\omega) \mathcal{D}_{\beta}+(\omega-r) \mathcal{L}_{\beta}+\omega \mathcal{U}_{\beta}\right] \\
& =\left(\mathcal{I}-r \mathcal{D}_{\beta}^{-1} \mathcal{L}_{\beta}\right)^{-1}\left[(1-\omega) \mathcal{I}+(\omega-r) \mathcal{D}_{\beta}^{-1} \mathcal{L}_{\beta}+\omega \mathcal{D}_{\beta}^{-1} \mathcal{U}_{\beta}\right] \\
& =(1-\omega) \mathcal{I}+\omega(1-r) \mathcal{D}_{\beta}^{-1} \mathcal{L}_{\beta}+\omega \mathcal{D}_{\beta}^{-1} \mathcal{U}_{\beta}+T_{\beta}
\end{aligned}
$$

where

$$
\begin{aligned}
T_{\beta}= & r \mathcal{D}_{\beta}^{-1} \mathcal{L}_{\beta}\left[(\omega-r) \mathcal{D}_{\beta}^{-1} \mathcal{L}_{\beta}+\omega \mathcal{D}_{\beta}^{-1} \mathcal{U}_{\beta}\right] \\
& +\left[r^{2}\left(\mathcal{D}_{\beta}^{-1} \mathcal{L}_{\beta}\right)^{2}+\cdots+r^{n-1}\left(\mathcal{D}_{\beta}^{-1} \mathcal{L}_{\beta}\right)^{n-1}\right] \times \\
& {\left[(1-\omega) \mathcal{D}_{\beta}^{-1}+(\omega-r)\left(\mathcal{D}_{\beta}^{-1} \mathcal{L}_{\beta}\right)+\omega\left(\mathcal{D}_{\beta}^{-1} \mathcal{U}_{\beta}\right)\right] \geq 0 }
\end{aligned}
$$

By similar arguments given in proof of (a), we can easily show that $\tilde{L}_{r w} \geq 0$. Since $0<\beta_{i} \leq 1$, it is obvious that $\mathcal{A}_{\beta}$ is an irreducible matrix. Thus $\tilde{L}_{r w}$ is a nonnegative irreducible matrix.

Theorem 3.2. Let $\mathcal{A} \in \mathbb{R}^{n \times n}$ be a nonsingular $L$-matrix. Assume that $0 \leq r \leq \omega \leq 1(r \neq 1, \omega \neq 0)$, and $0<\beta_{i} \leq 1$ but $\beta_{i-1} a_{i, i-1} \neq 0$ for some $i=1,2, \cdots, n-1$.

(a) If $\rho\left(L_{r \omega}\right)<1$, then $\rho\left(\tilde{L}_{r \omega}\right)<\rho\left(L_{r \omega}\right)<1$.

(b) If $\mathcal{A}$ is an irreducible matrix and if there exists a non-empty set $\alpha \subset Q=\{2,3, \cdots\}$ such that

$$
0<a_{i, i-1} a_{i-1, i}<1, \quad i \in \alpha, \quad \text { and } \quad a_{i, i-1} a_{i-1, i}=0, \quad i \in Q \backslash \alpha,
$$

then it holds that

$$
\left\{\begin{array}{lll}
\rho\left(\tilde{L}_{r \omega}\right)<\rho\left(L_{r \omega}\right) & \text { if } & \rho\left(L_{r \omega}\right)<1 ; \\
\rho\left(\tilde{L}_{r \omega}\right)=\rho\left(L_{r \omega}\right) & \text { if } & \rho\left(L_{r \omega}\right)=1 ; \\
\rho\left(\tilde{L}_{r \omega}\right)>\rho\left(L_{r \omega}\right) & \text { if } & \rho\left(L_{r \omega}\right)>1 .
\end{array}\right.
$$

Proof. Let

$$
\begin{aligned}
& M=\frac{1}{\omega}(\mathcal{I}-r \mathcal{L}), \\
& N=\frac{1}{\omega}[(1-\omega) \mathcal{I}+(\omega-r) \mathcal{L}+\omega \mathcal{U}), \\
& E_{\beta}=\frac{1}{\omega}\left(\mathcal{D}_{\beta}-r \mathcal{L}_{\beta}\right), \\
& F_{\beta}=\frac{1}{\omega}\left[(1-\omega) \mathcal{D}_{\beta}+(\omega-r) \mathcal{L}_{\beta}+\omega \mathcal{U}_{\beta}\right), \\
& M_{\beta}=\frac{1}{\omega}\left(\mathcal{I}+S_{\beta}\right)(\mathcal{I}-r \mathcal{L}), \\
& N_{\beta}=\frac{1}{\omega}\left(\mathcal{I}+S_{\beta}\right)[(1-\omega) \mathcal{I}+(\omega-r) \mathcal{L}+\omega \mathcal{U}) .
\end{aligned}
$$

Then we have the following splitting

$$
\mathcal{A}=M-N \quad \text { and } \quad \mathcal{A}_{\beta}=E_{\beta}-F_{\beta}=M_{\beta}-N_{\beta} .
$$


(a) Since $\mathcal{A}$ is an $L$-matrix and $0 \leq r \leq \omega \leq 1(r \neq 1, \omega \neq 0), M=$ $\frac{1}{\omega}(\mathcal{I}-r \mathcal{L})$ is a nonsingular $M$-matrix and $N \geq 0$ so that $\mathcal{A}=M-N$ is an $M$-splitting. By the fact that $\rho\left(L_{r w}\right)<1$ and Lemma 2.7, $\mathcal{A}$ is a nonsingular $M$-matrix. We also show that $\mathcal{A}_{\beta}$ is a nonsingular $M$ matrix using Lemma 2.9.

Since $\mathcal{A}_{\beta}$ is a nonsingular $M$-matrix, $\left(\mathcal{D}_{\beta}\right)_{i, i}>0$ and $D_{\beta}$ is invertible. Using the fact that $\left(\mathcal{L}_{\beta}\right)_{i j}=-a_{i j}+\beta_{i-1} a_{i, i-1} a_{i-1 j} \geq 0$, for $i=3, \cdots, n$, $j<i-2$, and $\left(\mathcal{L}_{\beta}\right)_{i, i-1}=-a_{i, i-1}\left(1-\beta_{i-1}\right) \geq 0, i=2, \cdots, n$, we have $\mathcal{L}_{\beta} \geq 0$ so that $E_{\beta}=\frac{1}{\omega}\left(\mathcal{D}_{\beta}-r \mathcal{L}_{\beta}\right)$ is a $Z$-matrix and it is also a nonsingular $M$-matrix. By our assumptions, $F_{\beta}$ is a nonnegative matrix so that $A_{\beta}=E_{\beta}-F_{\beta}$ is an $M$-splitting. Thus by Lemma 2.7, we have

$$
\rho\left(\tilde{L}_{r w}\right)=\rho\left(E_{\beta}^{-1} F_{\beta}\right)<1 .
$$

Using the fact that $\mathcal{A}=M-N, \mathcal{A}_{\beta}=E_{\beta}-F_{\beta}$ are $M$-splitting and $M^{-1} N=M_{\beta}^{-1} N_{\beta}$ yields that two splittings are regular and nonnegative.

On the other hand, $\mathcal{A}_{\beta}$ can be represented as

$$
\mathcal{A}_{\beta}=\left(\mathcal{I}+S_{\beta}\right) \mathcal{A}=\mathcal{I}-\mathcal{L}-\mathcal{U}+S_{\beta}-S_{\beta} \mathcal{L}-S_{\beta} \mathcal{U} .
$$

Denote by $S_{2}=S_{\beta} \mathcal{L}$. Then $S_{2}$ is a strictly lower triangular matrix. Let $S_{\beta} \mathcal{U}=S_{1}+S_{3}$ where $S_{1}$ and $S_{3}$ are diagonal and strictly upper triangular matrix of $S_{\beta} \mathcal{U}$, respectively. Then $\mathcal{D}_{\beta}=\mathcal{I}-S_{1}, \mathcal{L}_{\beta}=\mathcal{L}-S_{\beta}+S_{2}$, $\mathcal{U}_{\beta}=\mathcal{U}+S_{3}$, and

$$
\mathcal{A}_{\beta}=\mathcal{I}-\mathcal{L}-\mathcal{U}+S_{\beta}-S_{1}-S_{2}-S_{3}=\mathcal{D}_{\beta}-\mathcal{L}_{\beta}-\mathcal{U}_{\beta} .
$$

Note that

$$
\begin{aligned}
N_{\beta}-F_{\beta}== & \frac{1}{\omega}\left(\mathcal{I}+S_{\beta}\right)[(1-\omega) \mathcal{I}+(\omega-r) \mathcal{L}+\omega \mathcal{U}] \\
& \quad-\frac{1}{\omega}\left[(1-\omega) \mathcal{D}_{\beta}+(\omega-r) \mathcal{L}_{\beta}+\omega \mathcal{U}_{\beta}\right] \\
=\frac{1}{\omega} & {\left[(1-\omega)\left(\mathcal{I}-\mathcal{D}_{\beta}\right)+(\omega-r)\left(\mathcal{L}-\mathcal{L}_{\beta}\right)+\omega\left(\mathcal{U}-\mathcal{U}_{\beta}\right)\right.} \\
& \left.\quad+(1-\omega) S_{\beta}+(\omega-r) S_{2}+\omega\left(S_{1}+S_{3}\right)\right] \\
=\frac{1}{\omega} & {\left[(1-\omega)\left(\mathcal{I}-\mathcal{D}_{\beta}\right)+(\omega-r)\left(S_{\beta}-S_{2}\right)-\omega S_{3}\right.} \\
& \left.\quad+(1-\omega) S_{\beta}+(\omega-r) S_{2}+\omega\left(S_{1}+S_{3}\right)\right] \\
=\frac{1}{\omega} & {\left[(1-\omega)\left(\mathcal{I}-\mathcal{D}_{\beta}\right)+(1-r) S_{\beta}+\omega S_{1}\right] \geq 0 }
\end{aligned}
$$

Thus $N_{\beta} \geq F_{\beta}$ and $\mathcal{A}_{\beta}+N_{\beta} \geq \mathcal{A}_{\beta}+F_{\beta}$. Furthermore we have

$$
M_{\beta} \geq E_{\beta} \quad \text { and } \quad \mathcal{A}_{\beta}^{-1} M_{\beta} \geq \mathcal{A}_{\beta}^{-1} E_{\beta} \geq 0 .
$$


By Theorem 1.1 in [10], we have

$$
\rho\left(E_{\beta}^{-1} F_{\beta}\right) \leq \rho\left(M_{\beta}^{-1} N_{\beta}\right) .
$$

Hence

$$
\rho\left(\tilde{L}_{r w}\right)<\rho\left(L_{r w}\right)<1 .
$$

(b) Let $\mathcal{A}$ be an irreducible matrix. By Lemma $3.1, L_{r \omega}$ is a nonnegative and irreducible matrix, and by Lemma 2.5, there exists a positive vector $x$ such that

$$
L_{r \omega} \mathbf{x}=\lambda \mathbf{x}
$$

where $\lambda=\rho\left(L_{r w}\right)$. Thus we can easily show that

$$
[(1-\omega) \mathcal{I}+(\omega-r) \mathcal{L}+\omega \mathcal{U}] \mathbf{x}=\lambda(\mathcal{I}-r \mathcal{L}) \mathbf{x}
$$

or equivalently

$$
[(1-\omega-\lambda) \mathcal{I}+(\omega-r+r \lambda) \mathcal{L}+\omega \mathcal{U}] \mathbf{x}=0
$$

and

$$
(\lambda-1)(\mathcal{I}-r \mathcal{L}) \mathbf{x}=\omega(\mathcal{L}+\mathcal{U}-\mathcal{I}) \mathbf{x} .
$$

For the above $\lambda$ and $\mathbf{x}$ we have

$$
\begin{aligned}
\tilde{L}_{r w} \mathbf{x}- & \lambda \mathbf{x}= \\
= & \left(\mathcal{D}_{\beta}-r \mathcal{L}_{\beta}\right)^{-1}\left[(1-\omega) \mathcal{D}_{\beta}+(\omega-r) \mathcal{L}_{\beta}+\omega \mathcal{U}_{\beta}-\lambda\left(\mathcal{D}_{\beta}-r \mathcal{L}_{\beta}\right)\right] \mathbf{x} \\
= & \left(\mathcal{D}_{\beta}-r \mathcal{L}_{\beta}\right)^{-1}\left[(1-\omega-\lambda) \mathcal{D}_{\beta}+(\omega-r+r \lambda) \mathcal{L}_{\beta}+\omega \mathcal{U}_{\beta}\right] \mathbf{x} \\
= & \left(\mathcal{D}_{\beta}-r \mathcal{L}_{\beta}\right)^{-1}\left[(1-\omega-\lambda)\left(\mathcal{I}-S_{1}\right)+(\omega-r+r \lambda)\left(\mathcal{L}-S_{\beta}+S_{2}\right)\right. \\
+ & \left.\omega\left(\mathcal{U}+S_{3}\right)\right] \mathbf{x} \\
= & \left(\mathcal{D}_{\beta}-r \mathcal{L}_{\beta}\right)^{-1}\left[(\lambda+\omega-1) S_{1}+(\omega-r+r \lambda)\left(S_{2}-S_{\beta}\right)+\omega S_{3}\right] \mathbf{x} \\
= & \left(\mathcal{D}_{\beta}-r \mathcal{L}_{\beta}\right)^{-1}\left[(\lambda-1) S_{1}+\omega\left(S_{1}+S_{2}-S_{\beta}+S_{3}\right)+(\lambda r-r)\right. \\
& \left.\left(S_{2}-S_{\beta}\right)\right] \mathbf{x} \\
= & \left(\mathcal{D}_{\beta}-r \mathcal{L}_{\beta}\right)^{-1}\left[(\lambda-1) S_{1}+\omega\left(S_{\beta} \mathcal{U}+S_{\beta} \mathcal{L}-S_{\beta}\right)+r(1-\lambda)\right. \\
& \left.\left(S_{\beta}-S_{2}\right)\right] \mathbf{x} \\
= & \left(\mathcal{D}_{\beta}-r \mathcal{L}_{\beta}\right)^{-1}\left[(\lambda-1) S_{1}+\omega S_{\beta}(\mathcal{U}+\mathcal{L}-\mathcal{I})+r(1-\lambda)\left(S_{\beta}-S_{2}\right)\right] \mathbf{x} \\
= & \left(\mathcal{D}_{\beta}-r \mathcal{L}_{\beta}\right)^{-1}\left[(\lambda-1) S_{1}+(\lambda-1)(1-r) S_{\beta}\right] \mathbf{x},
\end{aligned}
$$

where $S_{1} \geq 0, S_{\beta} \geq 0$ and $1-r \geq 0$. Therefore

$$
\tilde{L}_{r w} \mathbf{x}-\lambda \mathbf{x}=(\lambda-1)\left(\mathcal{D}_{\beta}-r \mathcal{L}_{\beta}\right)^{-1}\left[S_{1}+(1-r) S_{\beta}\right] \mathbf{x} .
$$

If $\lambda<1$, then $\tilde{L}_{r w} \mathbf{x}-\lambda \mathbf{x} \leq 0$ and $\tilde{L}_{r w} \mathbf{x}-\lambda \mathbf{x} \neq 0$, i.e. $\tilde{L}_{r w} \mathbf{x} \leq \lambda \mathbf{x}$ and $\tilde{L}_{r w} \mathbf{x} \neq \lambda \mathbf{x}$. If $\lambda=1$, then $\tilde{L}_{r w} \mathbf{x}-\lambda \mathbf{x}=0$, i.e. $\tilde{L}_{r w} \mathbf{x}=\lambda \mathbf{x}$. If $\lambda>1$ then $\tilde{L}_{r w} \mathbf{x}-\lambda \mathbf{x} \geq 0$ and $\tilde{L}_{r w} \mathbf{x}-\lambda \mathbf{x} \neq 0$, i.e. $\tilde{L}_{r w} \mathbf{x} \geq \lambda \mathbf{x}$ and 
$\tilde{L}_{r w} \mathbf{x} \neq \lambda \mathbf{x}$. Using the above estimates and Lemma 2.6, we can easily prove the conclusion of (b) and it completes the proof.

It is well known that by taking a special value $\omega=r$ in AOR method, we obtain the SOR iteration. Hence we have the following result.

Corollary 3.3. Let $\mathcal{A} \in \mathbb{R}^{n \times n}$ be a nonsingular L-matrix. Assume that $0 \leq \omega \leq 1(\omega \neq 0)$ and $0<\beta_{i} \leq 1$ but $\beta_{i-1} a_{i, i-1} \neq 0$ for some $i=1,2, \cdots, n-1$.

(a) If $\rho\left(L_{\omega}\right)<1$, then $\rho\left(\tilde{L}_{\omega}\right)<\rho\left(L_{\omega}\right)<1$.

(b) If $\mathcal{A}$ is an irreducible matrix, and if there exists a non-empty set $\alpha \subset Q=\{2,3, \cdots, n\}$ such that

$$
0<a_{i, i-1} a_{i-1, i}<1, \quad i \in \alpha \quad \text { and } \quad a_{i, i-1} a_{i-1, i}=0, \quad i \in Q \backslash \alpha,
$$

then

$$
\left\{\begin{array}{lll}
\rho\left(\tilde{L}_{\omega}\right)<\rho\left(L_{\omega}\right) & \text { if } & \rho\left(L_{\omega}\right)<1 \\
\rho\left(\tilde{L}_{\omega}\right)=\rho\left(L_{\omega}\right) & \text { if } & \rho\left(L_{\omega}\right)=1 \\
\rho\left(\tilde{L}_{\omega}\right)>\rho\left(L_{\omega}\right) & \text { if } & \rho\left(L_{\omega}\right)>1
\end{array}\right.
$$

\section{The preconditioned AOR method for $H$-matrices}

In this section, we show the convergence of the preconditioned AOR method for the system (1) when the coefficient matrix $\mathcal{A}$ is an $H$-matrix. First, we give some lemmas which are useful in the sequel.

Lemma 4.1. [4] Let $\mathcal{A}$ be an $H$-matrix. Then $\left|\mathcal{A}^{-1}\right| \leq\langle\mathcal{A}\rangle$, where $\langle\mathcal{A}\rangle$ denotes the comparison matrix given in Definition 2 .

Lemma 4.2. [7] Let $\mathcal{A}$ and $\mathbf{B}$ be two $n \times n$ matrices with $0 \leq|\mathbf{B}| \leq \mathcal{A}$. Then $\rho(\mathbf{B}) \leq \rho(\mathcal{A})$.

By above lemmas we state and prove some lemmas and theorems.

Lemma 4.3. If $\mathcal{A}$ is a nonsingular $M$-matrix, then $\rho\left(L_{r \omega}\right)<1$.

Proof. Since $\mathcal{A}$ is an $M$-matrix, it is an $L$-matrix so that $M=\frac{1}{\omega}(\mathcal{I}-$ $r \mathcal{L})$ is a nonsingular $M$-matrix and $N=\frac{1}{\omega}[(1-\omega) \mathcal{I}(\omega-r) \mathcal{L}+\omega \mathcal{U}] \geq 0$. Hence $\mathcal{A}=M-N$ is an $M$-splitting, and by Lemma 2.7, $\rho\left(M^{-1} N\right)=$ $\rho\left(L_{r \omega}\right)<1$.

Lemma 4.4. If $\mathcal{A}$ is an $H$-matrix, then $\rho\left(L_{r \omega}\right)<1$. 
Proof. Let $\mathcal{A}$ be an $H$-matrix, then $\langle\mathcal{A}\rangle$ is an $M$-matrix. By Lemma 4.3, $\rho\left(\bar{L}_{r \omega}\right)<1$ where

$$
\bar{L}_{r \omega}=(I-r|L|)^{-1}[(1-\omega) I+(\omega-r)|L|+\omega|U|] .
$$

Let

$$
\begin{aligned}
& X=(I-r L)^{-1}, \\
& Y=(1-\omega) I+(\omega-r) L+\omega U, \\
& Z=(I-r|L|)^{-1}, \\
& T=(1-\omega) I+(\omega-r)|L|+\omega|U| .
\end{aligned}
$$

Obviously, $X$ is an $H$-matrix and $\langle X\rangle=Z$. By Lemma $4.1,\left|X^{-1}\right| \leq$ $Z^{-1}$. Hence, we have

$$
\left|X^{-1} Y\right| \leq\left|X^{-1}\right||Y| \leq\left|X^{-1}\right| T \leq Z^{-1} T
$$

and $\left|L_{r \omega}\right| \leq \bar{L}_{r \omega}$. From Lemma 4.2, we have $\rho\left(L_{r \omega}\right) \leq \rho\left(\bar{L}_{r \omega}\right)$ and this completes the proof.

Lemma 4.5. Let $\mathcal{A}$ be an $H$-matrix. Then

$$
\beta_{i}^{\prime}=1+\frac{\left|a_{i, i-1}\right|+1}{\mid a_{i, i-1}\left(2\left\|\langle\mathcal{A}\rangle^{-1}\right\|_{\infty}-1\right)}>1 .
$$

Proof. Since $\langle\mathcal{A}\rangle=\mathcal{I}-|\mathcal{L}|-|\mathcal{U}| \leq \mathcal{I}$ is a nonsingular $M$-matrix, $\langle\mathcal{A}\rangle^{-1} \geq 0$ and $0 \leq \mathcal{I} \leq\langle\mathcal{A}\rangle^{-1}$. Thus $\left\|\langle\mathcal{A}\rangle^{-1}\right\|_{\infty} \geq 1$ and then $\beta_{i}^{\prime}>1$.

Theorem 4.6. Let $\mathcal{A}$ be an $H$-matrix. Then $\mathcal{A}_{\beta}$ is an $H$-matrix and $\rho\left(\tilde{L}_{r \omega}\right)<1$ for $\beta_{i} \in\left[0, \beta_{i}^{\prime}\right), i=1,2, \cdots, n-1$.

Proof. Let $r=\langle\mathcal{A}\rangle^{-1} \mathbf{e}$ where $\mathbf{e}=(1,1, \cdots, 1)^{T}$. Since $\mathcal{A}$ is an $H$ matrix, by Theorem 2.8, there exists a vector $r>0$ such that $\langle\mathcal{A}\rangle r=$ e $>0$. We show that $\left\langle\mathcal{A}_{\beta}\right\rangle$ is an $M$-matrix. Note that

$$
\left(\left\langle\mathcal{A}_{\beta}\right\rangle r\right)_{1}=r_{1}-\sum_{j=2}^{n}\left|a_{1 j}\right| r_{j}=(\langle\mathcal{A}\rangle r)_{1}>0 .
$$


For $i=2, \cdots, n$, we have

$$
\begin{aligned}
\left(\left\langle\mathcal{A}_{\beta}\right\rangle\right)_{i}= & \left|1-\beta_{i-1} a_{i, i-1} a_{i-1, i}\right| r_{i}-\sum_{j \neq i}\left|a_{i j}-\beta_{i-1} a_{i, i-1} a_{i-1, j}\right| r_{j} \\
& \geq r_{i}-\beta_{i-1}\left|a_{i, i-1} a_{i-1, i}\right| r_{i}-\sum_{j \neq i, i-1}\left|a_{i j}\right| r_{j} \\
& -\sum_{j \neq i, i-1} \beta_{i-1}\left|a_{i, i-1} a_{i-1, j}\right| r_{j}-\left|a_{i, i-1}\right|\left|1-\beta_{i-1}\right| r_{i-1} \\
& \geq(\langle\mathcal{A}\rangle)_{i}+\left|a_{i, i-1}\right| r_{i-1}-\beta_{i-1}\left|a_{i, i-1}\right|\left[\left|a_{i-1, i}\right| r_{i}\right. \\
& \left.+\sum_{j \neq i, i-1}\left|a_{i-1 j}\right| r_{j}\right]-\left|a_{i, i-1}\right|\left|1-\beta_{i-1}\right| r_{i-1} \\
& =1+\left|a_{i, i-1}\right| r_{i-1}-\beta_{i-1}\left|a_{i, i-1}\right|\left[-(\langle\mathcal{A}\rangle)_{i}+r_{i-1}\right] \\
& -\left|a_{i, i-1}\right|\left|1-\beta_{i-1}\right| r_{i-1} \\
& =1+\left|a_{i, i-1}\right| r_{i-1}-\beta_{i-1}\left|a_{i, i-1}\right|\left[-1+r_{i-1}\right]-\left|a_{i, i-1}\right|\left|1-\beta_{i-1}\right| r_{i-1} \\
& =1+\beta_{i-1}\left|a_{i, i-1}\right|+\left[1-\beta_{i-1}-\left|1-\beta_{i-1}\right|\right]\left|a_{i, i-1}\right| r_{i-1} .
\end{aligned}
$$

If $0 \leq \beta_{i} \leq 1$, then

$$
\left(\left\langle\mathcal{A}_{\beta}\right\rangle r\right)_{i}=1+\beta_{i-1}\left|a_{i, i-1}\right|>0 .
$$

Therefore, $\left\langle\mathcal{A}_{\beta}\right\rangle$ is an $M$-matrix and $\mathcal{A}_{\beta}$ is an $H$-matrix. If $\beta_{i}>1$, then

$$
\begin{aligned}
\left(\left\langle\mathcal{A}_{\beta}\right\rangle r\right)_{i}= & 1+2\left|a_{i, i-1}\right| r_{i-1}-\left(2 r_{i-1}-1\right) \beta_{i}\left|a_{i, i-1}\right| \\
> & 1+2\left|a_{i, i-1}\right| r_{i-1}-\left(2 r_{i-1}-1\right)\left[1+\frac{\left|a_{i, i-1}\right|+1}{\left|a_{i, i-1}\right|\left(2\left\|\langle\mathcal{A}\rangle^{-1}\right\|_{\infty}-1\right)}\right] \\
& \left|a_{i, i-1}\right| \\
& \geq 1+\left|a_{i, i-1}\right|-\left(2\left\|\langle\mathcal{A}\rangle^{-1}\right\|_{\infty}-1\right)\left|a_{i, i-1}\right| \frac{\left|a_{i, i-1}\right|+1}{\left|a_{i, i-1}\right|\left(2\left\|\langle\mathcal{A}\rangle^{-1}\right\|_{\infty}-1\right)} \\
& =0 .
\end{aligned}
$$

Therefore, $\left\langle\mathcal{A}_{\beta}\right\rangle$ is an $M$-matrix and $\mathcal{A}_{\beta}$ is an $H$-matrix, and by Lemma 4.4, $\rho\left(\tilde{L}_{r \omega}\right)<1$.

For the SOR iterative method, the following corollary holds.

Corollary 4.7. Let $\mathcal{A}$ be an $H$-matrix. Then $\mathcal{A}_{\beta}$ is an $H$-matrix and $\rho\left(\tilde{L}_{\omega}\right)<1$ for $\beta_{i} \in\left[0, \beta_{i}^{\prime}\right), i=1,2, \cdots, n-1$. 


\section{Numerical experiments}

In this section, we give some numerical examples to show efficiency of the preconditioned AOR method.

Example 1. Suppose that the coefficient matrix $\mathcal{A}$ is as follows:

$$
\mathcal{A}=\left(\begin{array}{ccccc}
1 & -0.1 & -0.06 & -0.35 & -0.22 \\
-0.16 & 1 & -0.04 & -0.08 & -0.28 \\
-0.2 & -0.1 & 1 & -0.12 & -0.2 \\
-0.06 & -0.24 & -0.17 & 1 & -0.05 \\
-0.32 & -0.22 & -0.1 & -0.15 & 1
\end{array}\right)
$$

The coefficient matrix $\mathcal{A}$ is an $L$-matrix. Let $\beta_{1}=0.86, \beta_{2}=0.79$, $\beta_{3}=0.95$ and $\beta_{4}=0.92$. For $r=0.3093, \omega=0.9827$, we have $\rho\left(\tilde{L}_{r \omega}\right)=$ $0.5760<\rho\left(L_{r \omega}\right)=0.6107$, and for $r=\omega=0.66$ we get $\rho\left(\tilde{L}_{\omega}\right)=$ $0.6735<\rho\left(L_{\omega}\right)=0.6916$.

The matrix $\mathcal{A}$ is also an $H$-matrix. Let $\beta_{1}=3, \beta_{2}=2.6, \beta_{3}=4$ and $\beta_{4}=2$. For $r=0.3093$ and $\omega=0.9827$ we get $\rho\left(\tilde{L}_{r \omega}\right)=0.4892<$ $\rho\left(L_{r \omega}\right)=0.6107$ and for $r=\omega=0.85$ we get $\rho\left(\tilde{L}_{\omega}\right)=0.5009<\rho\left(L_{\omega}\right)=$ 0.5536 .

Example 2. Suppose that the coefficient matrix $\mathcal{A}$ is given by

$$
\mathcal{A}=\left(\begin{array}{ccccc}
1 & -\frac{1}{2 \times 10+1} & -\frac{1}{3 \times 10+1} & \cdots & -\frac{1}{n \times 10+1} \\
-\frac{1}{2 \times 10+2} & 1 & -\frac{1}{3 \times 10+2} & \cdots & -\frac{1}{n \times 10+2} \\
-\frac{1}{3 \times 10+3} & -\frac{1}{2 \times 10+3} & 1 & \cdots & -\frac{1}{n \times 10+3} \\
\vdots & \vdots & \vdots & \ddots & \vdots \\
-\frac{1}{n \times 10+n} & -\frac{1}{(n-1) \times 10+n} & -\frac{1}{(n-2) \times 10+n} & \cdots & 1
\end{array}\right)
$$

We take $\beta_{i}=0.98$ for all $i=1,2, \cdots, n-1$. Table 1 shows the spectral radii, $\rho\left(L_{r \omega}\right)$ and $\rho\left(\tilde{L}_{r \omega}\right)$, of AOR method and the preconditioned AOR method, respectively, for different values of $n$ and various $r$ and $\omega$. As this table shows, we have $\rho\left(L_{r \omega}\right)<\rho\left(\tilde{L}_{r \omega}\right)$ for each case.

Example 3. Suppose that the coefficient matrix $\mathcal{A}$ is as

$$
\mathcal{A}=\left(\begin{array}{ccccc}
1 & 0.2 & -0.2 & 0.2 & 0.1 \\
0.4 & 1 & 0.2 & -0.2 & 0.1 \\
-0.5 & 0.2 & 1 & 0.1 & -0.1 \\
0.3 & -0.6 & 0.3 & 1 & 0.1 \\
0.8 & 0.3 & -0.2 & 0.4 & 1
\end{array}\right)
$$


TABLE 1. Numerical results for Example 2.

\begin{tabular}{lllll}
\hline$n$ & $r$ & $\omega$ & $\rho\left(L_{r \omega}\right)$ & $\rho\left(\tilde{L}_{r \omega}\right)$ \\
\hline 50 & 0.45 & 0.78 & 0.3902 & 0.3799 \\
100 & 0.38 & 0.96 & 0.2903 & 0.2784 \\
150 & 0.37 & 0.96 & 0.3076 & 0.2971 \\
200 & 0.28 & 0.95 & 0.3399 & 0.3294 \\
\hline
\end{tabular}

The coefficient matrix $\mathcal{A}$ is an $H$-matrix. Let $\beta_{1}=0.99, \beta_{2}=0.8$, $\beta_{3}=0.56$ and $\beta_{4}=0.87$. For $r=0.35, \omega=0.98$, we have $\rho\left(\tilde{L}_{r \omega}\right)=$ $0.7533<\rho\left(L_{r \omega}\right)=0.7936$, and for $r=\omega=0.88$ we get $\rho\left(\tilde{L}_{\omega}\right)=$ $0.7043<\rho\left(L_{\omega}\right)=0.7323$.

Let $\beta_{1}=2.5, \beta_{2}=2.01, \beta_{3}=2.92$ and $\beta_{4}=2.21$. For $r=0.58, \omega=0.95$, we have $\rho\left(\tilde{L}_{r \omega}\right)=0.6346<\rho\left(L_{r \omega}\right)=0.7706$, and for $r=\omega=0.89$ we get $\rho\left(\tilde{L}_{\omega}\right)=0.6440<\rho\left(L_{\omega}\right)=0.7267$.

\section{References}

[1] Qingbing Liu, Guoliang Chen, Jing Cai, Convergence analysis of the preconditioned Gauss-Seidel method for $H$-matrices, Computer Math. Appl. 56 (2008) 2048-2053.

[2] T. Kohno, H. Kotakemori, H. Niki, M. Usui, Improving the Gauss-Seidel method for Z-matrices, Linear Algebra Appl., 267 (1997) 113-123.

[3] M.T. Darvishi, P. Hessari, B.-Y. Shin, Preconditioned modified AOR method for systems of linear equations, Comm. Numer. Meth. Engng., (in press), doi:10.1002/cnm.1330.

[4] A. Neumaier, On the comparison of $H$-matrices with $M$-matrices, Linear Algebra Appl. 83 (1986) 135-141.

[5] C. Li, D.J. Evans, Improving the SOR method, Techincal Report 901, Department of computer studies, University of Loughborough, 1994.

[6] A. Berman, R.J. Lemmons, Nonnegative matrices in the mathematics sciences, SIAM, Philadelphia, PA, 1994.

[7] R.S. Varga, Matrix iterative analysis, Prentice-Hall, Englewood Cliffs, NJ, 1962; Springer Series in Computational Mathematics, vol. 27, Springer-Verlag, Berlin, 2000.

[8] W. Li, W.W. Sun, Modified Gauss-Seidel type methods and Jacobi type methods for Z-matrices, Linear Algebra Appl. 317 (2000) 227-240.

[9] D.M. Young, Iterative solution for large linear systems, Academic press, New York, (1971).

[10] Y.Z. Song, Comparisons of nonnegative splitting of matrices, Linear Algebra Appl. 154-156 (1991) 433-455.

[11] Y.T. Li, S. Yang, A multi-parameters preconditioned AOR iterative method for linear systems, Appl. Math. Comput. 206 (2008) 465-473. 
${ }^{a}$ Department of Mathematics,

Chonnam National University,

South Korea

${ }^{b}$ Department of Mathematics,

Razi University, Kermanshah 67149,

Iran 